\section{G387(P) COMPARISON BETWEEN POINT OF CARE (POC) CRP ESTIMATION AND LABORATORY CRP VALUES IN PAEDIATRIC ASSESSMENT UNIT (PAU)}

B Saikia, J Srinivas. Paediatrics, Milton Keynes University Hospital NHS Trust, Milton Keynes, UK

\subsection{6/archdischild-2018-rcpch.376}

Background Point of care tests (PoCT), recent trend in healthcare helps provide rapid 'on site' results. These are considered to have potential to improve outcomes by optimising prescribing decisions, reducing referrals, improving efficiency of care and reducing length of stay in hospitals. CRP estimation is one of the PoCT done in children. There is however no published data for PoCT CRP performed in-hospital setting especially for children presenting acutely to paediatrics. A bedside CRP machine installed at our Paediatric Assessment Unit (PAU) provides result in 4 mins with one drop of blood. The machine has a range of CRP values between $5-200 \mathrm{mg} / \mathrm{L}$.

Aim To compare CRP values (PoCT vs Lab) in children presenting to PAU.

Method PoCT test was performed along with routine lab blood tests in children presenting to our PAU. This data was collected prospectively over 5 months between January and May 2017.

Results A total of100 paired CRP samples collected during that time. The children were aged between 1 month and 16 years. Of the 100 children, 35 had PoCT CRP $<5 \mathrm{mg} / \mathrm{L}$ and 14 children had value $>100 \mathrm{mg} / \mathrm{L}$. On analysis of the PoCT and lab CRP results, most of the values were within $\pm 2 \mathrm{SD}$ on a Bland Altman Plot.

Summary The above findings were presented at a joint paediatrics and pathology meeting in our Trust. Decision was made to continue PoCT CRP in our PAU as an alternative to lab testing. Medical and nursing staff found that the PoCT machine was reliable, easy to use and gave results within $4 \mathrm{~min}$. The other advantages are that this requires a small amount of blood $(1.5 \mu \mathrm{l})$ and quicker results compared to lab result. We found that those children who had CRP $<5 \mathrm{mg} / \mathrm{L}$ and clinically well were discharged promptly helping in improved flow in PAU. As staff sare increasingly confident of the results of this PoCT machine, this is now being used for serial CRP monitoring and as a reliable alternative to laboratory testing.

\section{G388(P) STAMP: A LONG TERM, ONGOING PRESCRIBING QI PROJECT TO IMPROVE PRESCRIBING AND MEDICATION SAFETY}

A Trivedi, K Styles, T Bate, R Ajitsaria. Paediatrics, The Hillingdon Hospitals NHS Foundation Trust, Uxbridge, UK

\subsection{6/archdischild-2018-rcpch.377}

Aim We describe an ongoing quality improvement project focusing on paediatric prescribing for medical, surgical and oncology patients in a district general hospital. The project is called STAMP - 'Safe Treatment and Administration of Medicine in Paediatrics'. The aim of the project was to identify why local errors were happening, improve feedback to prescribers when errors were made and to decrease the rate of these errors through multiple interventions.

Method Divisional lead pharmacist collected weekly error data for nine months. Individual feedback given to prescribers.
Error data shared with the paediatric team via departmental instant text message group.

Results On average 185 prescriptions reviewed per week. Mean error rate 5.2\% and worst error rate $12.6 \%$. The most common errors were incorrect or missing frequency and incorrect dose. There was an initial see-saw error rate and a period of improved prescribing in July and August 2017 which we felt was secondary to the teaching highlighting common errors.

However this improvement was not sustained. We noticed a decrease in errors when the ward was less busy which is consistent with prescribers reporting frequent interruptions and increased errors when there were new medical staff. As a result of feedback from prescribers we have made multiple interventions including

- sharing anonymous 'error of the week' examples on group text message service as a quiz with answer,

- new medication guideline for surgical and orthopaedic for their most frequently prescribed medications,

- departmental teaching, and

- prescribing station with prescribing resources to encourage prescribers to move to an interruption-free zone.

Conclusion Although we have not yet been able to demonstrate sustained improvements in prescribing error rates, we believe multiple small changes and a strong STAMP team are most likely to bring about a culture of safer prescribing within our department. We feel that our most significant steps so far are the teaching sessions and improved personal feedback to prescribers. We hope the new prescribing station will decrease interruptions during prescribing. We need to reach out to our junior surgical and orthopaedic colleagues who do not attend our teaching and may benefit from additional prescribing support.

\section{G389(P) TACKLING ENTRENCHED CULTURE AND PATIENT SAFETY ERROR THROUGH IMPROVEMENT AND EMPOWERMENT OF TRAINEES DURING MEDICAL HANDOVERS IN A PAEDIATRIC TEACHING HOSPITAL}

F Osborne, M Lister, S Joseph. Acute Receiving Unit, NHS Lothian, Royal Hospital for Sick Children, Edinburgh, UK

\subsection{6/archdischild-2018-rcpch.378}

\section{Aims}

- To improve the quality of information communicated at medical handovers to reduce the risk of patient safety errors.

- To empower junior trainees to lead medical handovers with the intention of improving patient ownership.

- To reduce inefficiencies during medical handovers and maximise efficiencies whilst delivering clinical care.

Methods Problems and ideas were brainstormed with the Acute Receiving Unit (ARU) team, identifying key themes and targets for intervention. A series of changes were implemented utilising 12 Plan Do Study Act (PDSA) cycles with collaborative team reviews of data to determine the next cycle intervention. Key interventions were: facilitating a junior-led handover supervised by consultants; providing guidance regarding model patient handovers; improved task allocating utilising 'mini teams' and the introduction of an active pause following the post-take handover to ask questions or discuss staff or services issues (named 'Robin's Pause'). Outcome 
measures included analysis of handover duration and a postimplementation staff survey to investigate staff perception and experience.

Results There is a sustained reduction in the length of the post-ward round handover from 94 to $35 \mathrm{~min}$ (10 days analysed pre and post-intervention). A qualitative survey found the following percentages of people agreed or strongly agreed that: job allocation is more robust and efficient (93\%), handover is more efficient (64\%) and there is improved patient 'ownership' (86\%). 93\% of respondents agreed that an active 'pause' at the end of the handover to ask questions or discuss staff and service issues was beneficial. The response rate was 14 out of 33 possible respondents (42\%). A key finding was that the respondents unanimously agreed that facilitating a junior-led handover was empowering for trainees and turned a service safety task into a training opportunity.

Conclusion Empowering junior members of the team to hand over patients in conjunction with a series of small changes improved the effectiveness and efficiency of the medical handover. It also enabled a cultural change as handover was seen as a training opportunity in addition to being a service function.

\section{G390(P) HOSPITAL AT NIGHT: OUR EXPERIENCES IN IMPROVING PATIENT CARE THROUGH ESTABLISHING A HAN TEAM}

M Lister, F Osborne, S Joseph. Acute Receiving Unit, Royal Hospital for Sick Children, Edinburgh, UK

\subsection{6/archdischild-2018-rcpch.379}

\section{Aims}

- To set up a Hospital At Night (HAN) team and provide the facilities for effective working.

- To improve communication within the HAN team.

- To reduce interruptions to handovers and maintain handovers within the allocated timeframe.

Background The decision to establish a HAN team was taken following review of the medical cover of a 130 bedded quaternary paediatric hospital. Key issues were:

- A lack of effective team working and inadequate communication leading to workload imbalances and risk of important tasks being missed.

- A poor escalation policy leading to inappropriate escalation of deteriorating patients and patient safety issues.

Methods To address the issues we set up a multidisciplinary working group comprising: a consultant paediatrician, a paediatric registrar, a nurse manager and a nurse co-ordinator. These individuals underwent a peer consultation programme and the working group then met with other HAN teams throughout the region to learn from their experiences. Several audits and 'time in motion' studies identified specific issues which could be addressed. 48 Plan Do Study Act (PDSA) cycles were undertaken over an 18 month period with close collaboration and communication between our project group, senior clinical management and front line teams.

Results Funding was obtained for a specifically adapted 'HAN Room' which has become the team base for handovers, administrative work and breaks. Improvements within this room included: improved IT facilities, lockers, basic catering facilities and increased space. An updated escalation policy for deteriorating patients has been implemented complemented with ward level teaching on identifying and managing a deteriorating patient for nursing teams. Interruptions to handovers have stopped and now 95\% of handovers occur within the allotted time. Variation from this occurs appropriately due to discussion about complex or deteriorating patients.

Conclusion We have set up a successful HAN team and provided it with appropriate facilities. By doing this we have significantly improved communication, handovers, team dynamics and the team culture. The hospital co-ordinator working within the HAN team now provides an excellent link between the HAN team and the ward nurses.

\section{G391(P) SCHOOL-BASED DIABETES CLINICS: QI TO ENGAGE FREQUENT NON-ATTENDERS AND IMPROVE TEENAGER'S SELF-MANAGEMENT}

\begin{abstract}
${ }^{1,2}{ }^{2}$ Alins Sahun, ${ }^{2} \mathrm{~K}$ Camara, ${ }^{2,3} \mathrm{~K}$ Gething, ${ }^{2} \mathrm{~J}$ Gane, ${ }^{2} \mathrm{D}$ Schenck, ${ }^{4,5} \mathrm{Y}$ Tse. ${ }^{1}$ Paediatric Diabetes Team, Plymouth Hospitals NHS Trust, Plymouth, UK; ${ }^{2}$ Paediatric Diabetes Team, The Great North Children's Hospital, Newcastle upon Tyne, UK; ${ }^{3}$ Clinical Psychology, The Great North Children's Hospital, Newcastle upon Tyne, UK; ${ }^{4}$ Quality Improvement Service, The Great North Children's Hospital, Newcastle upon Tyne, UK; ${ }^{5}$ Paediatric Renal Team, The Great North Children's Hospital, Newcastle upon Tyne, UK
\end{abstract}

\subsection{6/archdischild-2018-rcpch.380}

Background Optimising attendance rates in outpatient's clinics is important to ensure good clinical care and to avoid waste of scarce medical resources. In 2016, we identified a cohort of 17 teenagers that frequently Do Not Attend (DNA) the Paediatric Diabetes Multidisciplinary Clinics, compromising patient care and reducing compliance with NICE and BPT standards. As this cohort was found to be enrolled in a cluster of 5 schools we explored the benefit of taking diabetes clinics into school.

Objectives Design and deliver additional school-based clinics to facilitate teenager's engagement with healthcare services.

Patients and methods During 6 months quarterly school-based clinics were offered to all teenagers with T1DM in these secondary schools. A semi-structured interview was administered to identify their concerns about diabetes. Patients were invited to provide feedback about hospital clinics. 34 teenagers were targeted (17 initially identified as frequent non-attenders and 17 controls at same schools).

Results The hospital DNA rate was reduced by 50\%. HbA1c levels remained unchanged over the study period but school clinics allowed early identification of patients with rapid deterioration.School-based clinics improved teenagers' ability to express their real beliefs about diabetes and be actively involved in the management of their condition. This unveiled significant knowledge gaps not previously identified by the team in $50 \%$ of the participants. This clinic format improved communication and partnership with school nurses/health assistants, enabling healthcare plans to be more effectively carried out.Although patients were usually happy with hospital-based clinics, they complained about missing school, time spent in hospital and lack of privacy. Some teenagers perceived judgemental attitudes in healthcare professionals or parents.

Conclusions

- This pilot shows promise in improving contact in this difficult to reach group while reducing wasted hospital clinic slots.

- School clinics are an effective way to engage young people in self-management, actively preparing them for transition.

- A well powered trial may allow HbA1c improvement to be shown. 